\title{
On the Microscopic Validity of the Wulff Construction and of the Generalized Young Equation
}

\author{
Joël De Coninck, ${ }^{\star}$ François Dunlop and Vincent Rivasseau
}

Centre de Physique Théorique (CNRS-LP 14), Ecole Polytechnique, F-91128 Palaiseau Cedex, France

\begin{abstract}
For a large class of $1+1$ dimensional interfaces of the Solid-On-Solid type we prove on a microscopic basis the validity of the Wulff construction and of the generalized Young equation which gives the contact angle of a sessile drop on a wall. Our proof relies on a new method to treat random walks with a finite number of global constraints.
\end{abstract}

\section{Introduction}

Consider a phase $A$ in a container, whose walls are partially wet by droplets of a phase $B$. Although small, these droplets are macroscopic, and their contact angle $\Theta$ with the wall can be measured and studied as a function of temperature, concentration or any other parameters. A transition from partial wetting to complete wetting may occur, if the angle $\Theta$ decreases down to zero, where a thin film of the phase $B$ separates the phase $A$ from the wall.

It is well known that the contact angle $\Theta$ is related to the surface tension $\sigma_{A B}$ and wall free energies $\sigma_{A W}, \sigma_{B W}$ through Young's equation (Young 1805). For isotropic media, it reads

$$
\sigma_{A B} \cos \Theta=\sigma_{A W}-\sigma_{B W}
$$

The study of droplets and wetting films is also important in metals and other anisotropic media. There Young's equation has to be modified. It takes the form [1]

$$
\sigma_{A B}(\Theta, \varphi) \cos \Theta-\sin \Theta \frac{\partial}{\partial \Theta} \sigma_{A B}(\Theta, \varphi)=\sigma_{A W}-\sigma_{B W}
$$

Equation (2) is to be understood as follows: take a point anywhere on the borderline of the droplet. This corresponds to a choice of a direction $\varphi$ in the plane of the wall. The contact angle $\Theta=\Theta(\varphi)$ is then the angle of the wall with the tangent plane to the droplet at the given point. The function $\sigma_{A B}(\Theta, \varphi)$ is the $A-B$ interfacial free energy per unit area of a flat $A-B$ interface which would be parallel to the given tangent plane. Equation (2) now may be solved to give the

\footnotetext{
* On leave from: Faculte' des Sciences, Universite' de l'Etat B-7000 Mons, Belgium
} 
contact angle $\Theta(\varphi)$ in terms of $\sigma_{A B}(),. \sigma_{A W}, \sigma_{B W}$. Alternatively, this equation may be used to obtain information on the surface tension from measurements of the contact angle.

In statistical mechanics, in order to obtain macroscopic droplets and well defined contact angles, one must consider a statistical ensemble where the volume of one or several droplets is fixed and large. One should then prove that the droplet profile has relatively small fluctuations, and that the free energy is simply related to the integral of the surface tension over the mean profile. The equation for the mean profile, and the generalized Young equation (2), then follow by classical variational methods similar to those which yield the Wulff construction for the equilibrium shape of crystals $[2,3]$. The corresponding solutions may be obtained by a Wulff construction. The above program has been performed in [1] for the special case of the $1+1$ dimensional Gaussian model: the wall is one dimensional $(\mathbb{Z})$ and the height of the interface above the wall is a real random function, with a Gaussian distribution.

The present paper provides a proof of the Wulff construction and of the generalized Young equation for general $1+1$ dimensional models: the height of the $A B$ interface is a real random function, whose probability distribution comes from a general nearest neighbour coupling.

The paper is organized as follows. Section 2 is devoted to the mathematical question: "How to deal with random walks which are subject to a finite number of global constraints?" The physical applications are given in Sects. 3 and 4: We first study the shape of one droplet of length $N$ and $V \approx N^{2}$ as $N \rightarrow \infty$; this shape is shown to obey the Wulff construction. We then consider a random number of droplets on a wall of length $L$, in a canonical ensemble where the total volume of droplets is $V=\lambda L^{2}$ with $\lambda$ small. As $L \rightarrow \infty$, we prove that the volume concentrates in one droplet, whose contact angle obeys the generalized Young equation (2).

\section{Random Walks with Global Constraints}

We consider one droplet of two dimensional volume $V$ and length $N \approx V^{1 / 2}$ on a wall; the shape of the droplet is given by $\left(h_{0}, h_{1}, \ldots, h_{N}\right)$ with the constraints

$$
h_{0}=h_{N}=0, \quad h_{i}>0, \quad i=1, \ldots, N-1, \quad \sum_{0}^{N} h_{i}=V .
$$

The corresponding probability distribution is given by

where

$$
Z_{V, N}^{-1} \exp \left(-\sum_{1}^{N} e\left(h_{i}-h_{i-1}\right)\right) \delta\left(h_{0}\right) \delta\left(h_{N}\right) \delta\left(\Sigma h_{i}-V\right) \prod d h_{i}
$$

$$
Z_{V, N}=\int_{0}^{\infty} d h_{0} \int_{0}^{\infty} d h_{1} \cdots \int_{0}^{\infty} d h_{N} \exp \left(-\sum_{1}^{N} e\left(h_{i}-h_{i-1}\right)\right) \delta\left(h_{0}\right) \delta\left(h_{N}\right) \delta\left(\Sigma h_{i}-V\right),
$$

and the coupling $e(x)$ is a continuous even function strictly increasing for $x>0$, and such that

$$
\lim _{x \rightarrow \infty} x^{-1} e(x)=\infty
$$


or

$$
\left\{\begin{array}{l}
\lim _{x \rightarrow \infty} x^{-1} e(x)=a_{0} \quad \text { and } \\
\limsup _{x \rightarrow \infty}(\log x)^{-1}\left(e(x)-a_{0} x\right)<\infty
\end{array}\right.
$$

Examples for $e(x)$ are $|x|$ (continuous SOS model), $x^{2}$ (Gaussian), $\left(1+x^{2}\right)^{1 / 2}$ (arc length). Clearly the variables $x_{i}=h_{i}-h_{i-1}$ are only coupled through the constraints. Omitting for the moment the constraints $h_{i} \geqq 0$, we get that the $x_{i}$ 's are distributed according to

$$
\exp \left(-\sum_{1}^{N} e\left(x_{i}\right)\right) \delta\left(\Sigma x_{i}\right) \delta\left(\Sigma(N-i) x_{i}-V\right) \prod d x_{i}
$$

For this fixed length $N$, we would like to study first the most probable droplet according to (4).

The difficulty is of course to take into account the constraints $\sum_{i} x_{i}=0$ and $\sum_{i}(N-i) x_{i}=V$. Two main ideas will be used: first, the Dirac form of the constraints allows us to introduce Lagrange multipliers in the Boltzmann factor, without changing the probability distribution. This gives a guess for the mean shape of the droplet, where each $\left\langle x_{j}\right\rangle$ is obtained, independently of the other $x_{i}$ 's, from the energy function $e(\cdot)$ and from $j, N, V$. The second idea is to keep the adjusted Lagrange multiplier and omit temporarily the Dirac constraints, and to use a suitably generalized version of the local central limit Theorem (Lemma 4 in the appendix). In this way, we obtain the joint probability distribution of any two increments $x_{j}$ and $x_{j^{\prime}}$, together with the appropriate constraint variables:

$$
\begin{aligned}
R_{j j^{\prime}} & =N^{-1 / 2} \sum_{i \neq j, j^{\prime}}\left(x_{i}-\left\langle x_{i}\right\rangle\right), \\
V_{j j^{\prime}} & =N^{-1 / 2} \sum_{i \neq j, j^{\prime}}(1-2 i / N)\left(x_{i}-\left\langle x_{i}\right\rangle\right) .
\end{aligned}
$$

Putting back the Dirac constraints yields the desired joint probability distribution of $y_{j}$ and $y_{j^{\prime}}$. This is the content of the following theorem:

Theorem 1. Let $h_{0}, h_{1}, \ldots, h_{N}$ be real random variables distributed according to

$$
\tilde{Z}_{V, N}^{-1} \exp \left(-\sum_{1}^{N} e\left(h_{i}-h_{i-1}\right)\right) \delta\left(h_{0}\right) \delta\left(h_{N}\right) \delta\left(\sum_{i} h_{i}-V\right) \prod d h_{i}
$$

where

$$
\tilde{Z}_{V, N}=\int_{-\infty}^{+\infty} d h_{0} \int_{-\infty}^{+\infty} d h_{1} \cdots \int_{-\infty}^{+\infty} d h_{N} \exp \left(-\sum_{1}^{N} e\left(h_{i}-h_{i-1}\right)\right) \delta\left(h_{0}\right) \delta\left(h_{N}\right) \delta\left(\Sigma h_{i}-V\right),
$$

and the coupling e(x) satisfies $(5 a)$ or $(5 b)$. Let

$$
\begin{aligned}
& t(c)=\int x e^{-e(x)+c x} d x / \int e^{-e(x)+c x} d x, \\
& I(c)=\frac{1}{2} \int_{0}^{c} c^{\prime} t\left(c^{\prime}\right) d c^{\prime},
\end{aligned}
$$

and let $c_{\lambda}$ be the solution of $c^{-2} I(c)=\lambda$. 
Let $V=\lambda N^{2}$. Then, as $N \rightarrow \infty$, the random variables $h_{1}-h_{0}, h_{2}-h_{1} \ldots$, $h_{N}-h_{N-1}$ are asymptotically distributed according to

$$
e^{-e\left(h_{j}-h_{J}-1\right)+c_{\lambda}(1-2 J / N)\left(h_{j}-h_{J}-1\right)} d\left(h_{j}-h_{j-1}\right),
$$

so that

$$
\begin{gathered}
\left\langle h_{j}-h_{j-1}\right\rangle_{N}=t\left(\left(1-\frac{2 j}{N}\right) c_{\lambda}\right)+O\left(N^{-1}\right), \\
\left\langle h_{j}\right\rangle_{N}=N \int_{(1-2 j / N) c_{\lambda}}^{c_{j}} t(c) d c+O(1), \\
\left\langle\left(h_{j}-h_{j-1}\right) ;\left(h_{j^{\prime}}-h_{j^{\prime}-1}\right)\right\rangle_{N}=O\left(N^{-1}\right) \quad \text { if } j \neq j^{\prime}, \\
\left\langle h_{j} ; h_{j^{\prime}}\right\rangle=O(N) \quad \forall j, j^{\prime},
\end{gathered}
$$

where $\langle\cdot\rangle_{N}$ is the mean value of $\cdot$ with respect to the probability distribution induced by $(6)$, and $\langle\because\rangle$ denotes truncated expectation values.

Proof: We first replace (6) by the equivalent expression (thanks to the Dirac measures):

$$
\begin{aligned}
& \tilde{Z}_{V, N}^{-1} \exp \left(-\sum_{1}^{N}\left[e\left(x_{i}\right)+c\left(1-\frac{2 i}{N}\right)\left(x_{i}-t\left[\left(1-\frac{2 i}{N}\right) c\right]\right)\right]\right) \\
& \delta\left(\Sigma x_{i}\right) \delta\left(\Sigma\left(\frac{N}{2}-i\right) x_{i}-V\right) \prod d x_{i}
\end{aligned}
$$

and we adjust $c$ so that the constraints will be satisfied in the average by the decoupled measure (i.e. without constraints), whose expectations will be denoted \langle\rangle$_{0}(c)$. For any $c$, we have

$$
\left\langle x_{j}\right\rangle_{0}(c)=-\left\langle x_{N-j}\right\rangle_{0}(c) \geqq 0 \quad \text { for } \quad 0<j \leqq \frac{N}{2},
$$

which implies

$$
\left\langle\Sigma x_{i}\right\rangle_{0}(c)=0
$$

Let us now consider the volume constraint:

$$
\begin{aligned}
-\sum_{i=1}^{N} i\left\langle x_{i}\right\rangle_{0}(c) & =\sum_{i=1}^{N}\left(\frac{N}{2}-i\right)\left\langle x_{i}\right\rangle_{0}(c) \\
& =\frac{N^{2}}{2 c^{2}} \sum_{j=1}^{[N / 2]}\left(\frac{2 j}{N} c\right)\left(\frac{2 c}{N}\right) t\left(\frac{2 j}{N} c\right) \equiv \frac{N^{2}}{c^{2}} I_{N}(c) .
\end{aligned}
$$

Remark that $I_{N}(c)$ is the Riemann sum approximation of $I(c)$. Lemma 3 therefore allows us to choose

$$
c_{N}=c\left(\frac{V}{N^{2}}, N\right) \simeq c\left(\frac{V}{N^{2}}, \infty\right)=c(\lambda, \infty) \equiv c_{\lambda},
$$


so that

$$
I_{N}\left(c_{N}\right)=\frac{c_{N}^{2} V}{N^{2}}
$$

(For example, in the purely gaussian case, one finds $c_{N}=6 \mathrm{~V} / \mathrm{N}^{2}\left\langle x^{2}\right\rangle_{0}(0)$ ). Returning to the general case, we see that this choice of $c_{N}$ gives moments $\left\langle\left(x_{j}\right)^{k}\right\rangle_{0}\left(c_{N}\right)$ bounded uniformly in $j$ and $N$, for any given $k$, and any given value of $V / N^{2}$. Now let

$$
t_{j N}=\left\langle x_{j}\right\rangle_{0}\left(c_{N}\right)=t\left(\left(1-\frac{2 j}{N}\right) c_{N}\right), \quad x_{j}=t_{j N}+y_{j}
$$

The $y_{j}$ 's are distributed according to

$$
\exp \left(-\sum_{1}^{N} e\left(t_{i N}+y_{i}\right)+\sum_{i}^{N} c_{N}\left(1-\frac{2 i}{N}\right) y_{i}\right) \delta\left(\Sigma y_{i}\right) \delta\left(\Sigma i y_{i}\right) .
$$

We shall now use Lemma 4 (cf. Appendix) to estimate the fluctuations with the constraints. For any given $j, j^{\prime}$ consider the 4 random variables

$$
y_{j}, y_{j^{\prime}}, \quad R_{i j^{\prime}}=N^{-1 / 2} \sum_{i \neq j, j^{\prime}} y_{i}, \quad V_{j j^{\prime}}=N^{-1 / 2} \sum_{i \neq j, j^{\prime}}\left(1-\frac{2 i}{N}\right) y_{i} .
$$

We wish to apply Lemma 4 with $\vec{Y}_{n}=\left(R_{j j^{\prime}}, V_{j j^{\prime}}\right)$, and we first compute the matrix $\Gamma_{n}$. We have

$$
\left\langle y_{j}^{2}\right\rangle_{0}\left(c_{N}\right)=\frac{\int d y y^{2} e^{-e\left(t_{J N}+y\right)+(1-2 j / N) c_{N} y}}{\int d y e^{-e\left(t_{J N}+y\right)+(1-2 j / N) c_{N} y}} .
$$

Since $t_{j N}=t\left((1-2 j / N) c_{N}\right)$, we may write

$$
\left\langle y_{j}^{2}\right\rangle_{0}\left(c_{N}\right)=q^{2}\left(\left(1-\frac{2 j}{N}\right) c_{N}\right) \simeq q^{2}\left(\left(1-\frac{2 j}{N}\right) c_{\lambda}\right) .
$$

Therefore

$$
\begin{aligned}
\left\langle R_{j j^{\prime}}^{2}\right\rangle_{0}\left(c_{N}\right) & =N^{-1} \sum_{i \neq j, j^{\prime}}\left\langle y_{i}^{2}\right\rangle_{0}\left(c_{N}\right)=N^{-1} \sum_{i \neq j, j^{\prime}} q^{2}\left(\left(1-\frac{2 i}{N}\right) c_{N}\right) \\
& =c_{N}^{-1} \sum_{1}^{[N / 2]} \frac{2 c_{N}}{N} q^{2}\left(\left(1-\frac{2 i}{N}\right) c_{N}\right)+O\left(N^{-1}\right) \simeq c_{\lambda}^{-1} \int_{0}^{c_{1}^{\prime}} d c^{\prime} q^{2}\left(c^{\prime}\right) .
\end{aligned}
$$

Similarly

$$
\begin{aligned}
\left\langle V_{j j^{\prime}}^{2}\right\rangle_{0}\left(c_{N}\right) & =N^{-1} \sum_{i \neq j, j^{\prime}}\left\langle\left(1-\frac{2 i}{N}\right)^{2} y_{i}^{2}\right\rangle \\
& =c_{N}^{-3} \sum_{1}^{[N / 2]}\left(\left(1-\frac{2 i}{N}\right) c_{N}\right)^{2} \frac{2 c_{N}}{N} q^{2}\left(\left(1-\frac{2 i}{N}\right) C_{N}\right)+O\left(N^{-1}\right) \\
& \simeq c_{\lambda}^{-3} \int_{0}^{c} c^{\prime 2} q^{2}\left(c^{\prime}\right) d c^{\prime}
\end{aligned}
$$


and, by symmetry,

$$
\left\langle R_{j j^{\prime}} V_{j j^{\prime}}\right\rangle_{0}\left(c_{N}\right)=N^{-1} \sum_{i \neq j, j^{\prime}}\left\langle\left(1-\frac{2 i}{N}\right) y_{i}^{2}\right\rangle_{0}\left(c_{N}\right)=O\left(N^{-1}\right),
$$

where $O\left(N^{-1}\right)$ comes from $i=N-j$ and $i=N-j^{\prime}$ whose symmetric terms are missing.

We have thus proven that the matrices $\Gamma_{N}$ converge. In order to apply Lemma 4, we can't just take $X_{i N}=\left(y_{i},(1-2 i / N) y_{i}\right)$ which doesn't have a density with respect to the Lebesgue measure on $\mathbb{R}^{2}$ (the ratio of the two components is fixed). Instead we choose (for $N$ even, but the proof is easily adapted to $N$ odd):

$$
\begin{gathered}
\vec{X}_{i N}=\left[\begin{array}{l}
y_{1}+y_{i+N / 2} \\
\left(1-\frac{2 i}{N}\right) y_{i}-\frac{2 i}{N} y_{i+N / 2}
\end{array}\right] \begin{array}{l}
i=2,3, \ldots, N / 2 \\
i \neq j, j^{\prime}, N-j, N-j^{\prime},
\end{array} \\
\vec{X}_{1 N}=\left[\begin{array}{l}
y_{1}+y_{1+N / 2}+y_{j \pm N / 2}+y_{j^{\prime} \pm N / 2} \\
\left(1-\frac{2}{N}\right) y_{1}-\frac{2}{N} y_{1+N / 2}-\left(1-\frac{2(j \pm N / 2)}{N}\right) y_{j \pm N / 2}-\left(1-\frac{2\left(j^{\prime} \pm N / 2\right)}{N}\right) y_{j^{\prime} \pm N / 2}
\end{array}\right],
\end{gathered}
$$

where the + or - signs are chosen so that $0 \leqq j \pm N / 2 \leqq N$ and $0 \leqq j^{\prime} \pm N / 2 \leqq N$. The hypotheses of Lemma 4 are then easily verified, for any $k$ and $\delta$. We take $k=4$, but discard the $k=4$ correction term into an $O\left(N^{-1}\right)$. Incorporating the constraints, we find that $y_{j}$ and $y_{j^{\prime}}$ have asymptotically a joint distribution proportional to

$$
\begin{aligned}
& e^{-\tilde{e}_{,}\left(i_{j}\right)-\tilde{e}_{j},\left(y_{j^{\prime}}\right)} f_{N}\left(-N^{-1 / 2}\left(y_{j}+y_{j^{\prime}}\right),-N^{-1 / 2}\left[\left(1-\frac{2 j}{N}\right) y_{j}+\left(1-\frac{2 j^{\prime}}{N}\right) y_{j^{\prime}}\right]\right) \\
& \approx e^{-\tilde{e}_{j}\left(y_{j}\right)-\tilde{e}_{j}\left(y_{j^{\prime}}\right)}\left\{e^{-N^{-1}\left(a_{j} y_{j}^{2}+a_{j}, y_{j^{\prime}}^{2}+b y_{j} y_{j^{\prime}}\right)} .\right. \\
& \left.\quad \cdot\left(1+N^{-1 / 2} P_{2}\left(N^{-1 / 2} y_{j}, N^{-1 / 2} y_{j^{\prime}}\right)\right)+O\left(N^{-1}\right)\right\},
\end{aligned}
$$

where

$$
\tilde{e}_{j}\left(y_{j}\right)=e\left(t_{j N}+y_{j}\right)-\left(1-\frac{2 j}{N}\right) c_{N} y_{j},
$$

and $a_{j}, a_{j^{\prime}}$ and $b$ have a limit as $N \rightarrow \infty$, and $P_{2}$ is a quadratic polynomial whose coefficients converge as $N \rightarrow \infty$. This implies the desired estimates

$$
\left\langle y_{j}\right\rangle=O\left(N^{-1}\right),\left\langle y_{j}^{2}\right\rangle=O(1),\left\langle y_{j} y_{j^{\prime}}\right\rangle=O\left(N^{-1}\right) .
$$

which proves (10) and (11) of Theorem 1, where $O\left(N^{-1}\right)$ also includes the error due to the difference between $c_{N}$ and $c_{\lambda}$ (Riemann sum approximation). The other statements in the theorem follow easily. We now take into account the wall condition $h_{i} \geqq 0$ :

Corollary. Under the hypotheses of Theorem 1 , suppose that $h_{1} \cdots h_{N-1}$ are restricted to be positive and denote the corresponding expectation values by $\langle\cdot\rangle_{N, h}>0$. Then 
$\exists a, b, \zeta>0$ such that and

$$
\left|\left\langle h_{j}-h_{j-1}\right\rangle_{N, h>0}-\left\langle h_{j}-h_{j-1}\right\rangle_{N}\right|<O\left(N^{-1}\right)+a \cdot \exp (-b \operatorname{Min}(j, N-j))
$$

$$
Z_{V, N} / \tilde{Z}_{V, N} \rightarrow \zeta \quad \text { as } N \rightarrow \infty .
$$

Remark. Brownian motion conditioned to be positive has been studied in particular in [7]. The present Corollary deals with a rather different situation, because the volume constraint by itself tends to push the walk away from the wall.

Proof. The method of proof is adapted from [1] (Gaussian model). Let

$$
\begin{gathered}
\chi_{h_{i}>0}=\left\{\begin{array}{lll}
1 & \text { if } & h_{i}>0 \\
0 & \text { if } & h_{i} \leqq 0
\end{array}, \quad \chi_{h_{i} \leq 0}=1-\chi_{h_{1}>0},\right. \\
\zeta_{N}=\left\langle\prod_{i} \chi_{h_{\mathrm{t}}>0}\right\rangle_{N}=Z_{V . N} / \tilde{Z}_{V, N}, \quad \zeta_{N}^{0}=\left\langle\prod_{i} \chi_{h_{\mathrm{r}}>0}\right\rangle_{0}\left(c_{N}\right) .
\end{gathered}
$$

Since $\zeta_{N}^{0}$ is associated to a random walk with independent steps, we know that

$$
\zeta_{N}^{0} \rightarrow \zeta>0 \text { as } \quad N \rightarrow \infty .
$$

Let us now prove the following lemma:

Lemma 1. $\exists b>0$ such that $\forall N, i$ with $1 \leqq i \leqq N-1$,

$$
\left\langle\chi_{h_{1} \leq 0}\right\rangle_{N} \leqq \exp (-b \operatorname{Min}(i, N-i)) \text {. }
$$

Proof. We begin as usual (e.g. [6], p. 52):

$$
\left\langle\chi_{h_{1} \leq 0}\right\rangle_{N} \leqq\left\langle\exp \left(-t h_{i}\right)\right\rangle_{N}=\exp \left(-t\left\langle h_{i}\right\rangle_{0}\left(c_{N}\right)\right) \cdot\left\langle\exp \left(-t \sum_{1}^{i} y_{j}\right)\right\rangle_{N} \text {. }
$$

We then study $\sum_{1}^{i} y_{j}$ together with the constraint variables as in Theorem 1, and obtain

$$
\left\langle\exp \left(-t \sum_{1}^{i} y_{j}\right)\right\rangle_{N} \leqq \exp \left(\left(b_{1} t / N+b_{2} t^{2}\right) \operatorname{Min}(i, N-i)\right)
$$

from which the lemma follows by an optimal choice of $t$. For any $N_{0} \ll N$, we then have

$$
\begin{aligned}
\zeta_{N} & =\left\langle\prod_{i}^{N_{0}-1} \chi_{h_{1}>0} \cdot \prod_{N-N_{0}+1}^{N-1} \chi_{h_{i}>0} \cdot \prod_{N_{0}}^{N-N_{0}}\left(1-\chi_{h_{i} \leq 0}\right)\right\rangle_{N} \\
& \geqq\left\langle\prod_{i}^{N_{0}-1} \chi_{h_{l}>0} \cdot \prod_{N-N_{0}+1}^{N-1} \chi_{h_{i}>0}\right\rangle_{N}-\sum_{N_{0}}^{N-N_{0}}\left\langle\chi_{h_{i} \leq 0}\right\rangle_{N} \\
& \geqq\left\langle\prod_{i}^{N_{0}-1} \chi_{h_{i}>0} \cdot \prod_{N-N_{0}+1}^{N-1} \chi_{h_{i}>0}\right\rangle_{N}-\frac{2 \exp \left(-b N_{0}\right)}{1-\exp (-b)}
\end{aligned}
$$

We want to prove $\zeta_{N} \rightarrow \zeta$. Given $\varepsilon>0$, we fix $N_{0}$ so that the last term is less than $\varepsilon / 2$. Because $N_{0}$ is fixed, we can again apply the method of Theorem 1 to show 
that the first term equals the same in the measure $\langle\cdot\rangle_{0}\left(c_{N}\right)$, plus an error $O\left(N_{0} / N\right)$. This proves the second half of the corollary. Similarly we have

$$
\left\langle h_{j}-h_{j-1}\right\rangle_{N, h>0}=\frac{\left\langle\left(h_{j}-h_{j-1}\right) \prod_{1}^{N-1} \chi_{h_{1}>0}\right\rangle_{N}}{\left\langle\prod_{1}^{N-1} \chi_{h_{i}>0}\right\rangle_{N}}=t_{j N}+\frac{\left\langle y_{j} \prod_{1}^{N-1} \chi_{h_{l}>0}\right\rangle_{N}}{\left\langle\prod_{1}^{N-1} \chi_{h_{1}>0}\right\rangle_{N}},
$$

so that

$$
\begin{aligned}
\left|\left\langle h_{j}-h_{j-1}\right\rangle_{N, h>0}-\left\langle h_{j}-h_{j-1}\right\rangle_{N}\right| \leqq & O\left(N^{-1}\right)+O\left(\exp \left(-b N_{0}\right)\right)+\zeta_{N}^{-1} \\
& -\left\langle y_{j} \prod_{1}^{N_{0}-1} \chi_{h_{l}>0} \cdot \prod_{N-N_{0}+1}^{N-1} \chi_{h_{i}>0}\right\rangle_{N} .
\end{aligned}
$$

Given any $\varepsilon>0$, we should prove that this is less than

$$
c_{1} N^{-1}+c_{2} \exp (-b j)+\varepsilon
$$

for all $j$ and $N$ with $j \leqq N / 2$. We fix $N_{0}$ so that $O\left(\exp \left(-b N_{0}\right)\right)<\varepsilon$. Then we analyze the last term in the measure $\langle\cdot\rangle_{0}\left(c_{N}\right)$ together with the constraint variables as in Theorem 1. If $N_{0} \leqq j \leqq N-N_{0}$, this last term is $O\left(N^{-1}\right)$. If $j<N_{0}$, then we get an estimate $a \cdot \exp (-b j)$ in the measure $\langle\cdot\rangle_{0}\left(c_{N}\right)$ plus an error $O\left(N^{-1}\right)$ from the constraints. This concludes the proof of the Corollary.

\section{The Wulff Construction}

We now present in this section how the previous ideas and results may be used to establish the validity of the Wulff construction for a single droplet of length $N$ and "volume" $V=\lambda N^{2}$.

Let us indeed estimate the partition function

$$
\begin{aligned}
\tilde{Z}_{V, N} & =\int_{-\infty}^{+\infty} d h_{0} \cdots \int_{-\infty}^{+\infty} d h_{N} \exp \left(-\sum_{1}^{N} e\left(h_{i}-h_{i-1}\right)\right) \delta\left(h_{0}\right) \delta\left(h_{N}\right) \delta\left(\Sigma h_{i}-V\right) \\
& =\tilde{Z}_{V, N}^{0}\left(c_{N}\right)\left\langle\delta\left(\sum_{i} y_{i}\right) \delta\left(\Sigma\left(1-\frac{2 i}{N}\right) y_{i}\right)\right\rangle_{0}\left(c_{N}\right),
\end{aligned}
$$

where $c_{N} \approx c_{\lambda}$ has been defined after Eq. (12). Using Theorem 1, we get

$$
\log \tilde{Z}_{V, N}=\log \tilde{Z}_{V, N}^{0}\left(c_{N}\right)+O(\log N),
$$

and the decoupled partition function may be calculated:

with

$$
\log \tilde{Z}_{V, N}^{0}\left(c_{N}\right)=\sum_{i=1}^{N} \log \tilde{z}^{0}\left(\left(1-\frac{2 i}{N}\right) c_{N}\right)
$$

Denoting

$$
\tilde{z}^{0}(c)=\int e^{-e(t(c)+y)+c y} d y .
$$

$$
\tan \Theta(i)=t\left(\left(1-\frac{2 i}{N}\right) c_{N}\right),
$$


and comparing with $[1$, Theorem 1$]$, we see that

$$
-\log \tilde{z}^{0}\left(\left(1-\frac{2 i}{N}\right) c_{N}\right)=\frac{\sigma_{A B}(\Theta(i))}{\cos \Theta(i)},
$$

and therefore asymptotically

$$
-\log \tilde{Z}_{V, N}^{0}\left(c_{N}\right) \simeq \int_{0}^{N} \sigma_{A B}(\Theta(x)) \frac{d x}{\cos \Theta(x)} \simeq \int_{\text {mean profile }} \sigma_{A B}(\Theta(l)) d l .
$$

Using the Corollary to relate $Z_{V, N}$ and $\tilde{Z}_{V, N}$, we thus have:

Theorem 2. Let $h_{0} \cdots h_{N}$ be real random variables distributed according to

$$
Z_{V, N}^{-1} \exp \left(-\sum_{1}^{N} e\left(h_{i}-h_{i-1}\right)\right) \delta\left(h_{0}\right) \delta\left(h_{N}\right) \delta\left(\sum_{i} h_{i}-V\right) \prod_{i} d h_{i},
$$

where $Z_{V, N}$ is a normalization factor and $V=\lambda N^{2}$. If e $(\cdot)$ verifies hypothesis $(5 a)$ or $(5 b)$, then

$$
\frac{1}{N} \log Z_{V . N}=\frac{1}{N} \int_{I_{A B}} \sigma_{A B}(\Theta(l)) d l+O\left(\frac{\log N}{N}\right),
$$

where $I_{A B}$ is the mean profile.

This additivity of the free energy associated to the interface $A B$ is a central result in our analysis. It will be an essential ingredient in order to establish the microscopic validity of the Wulff construction.

Proposition 1. Let a sessile drop on a wall be described by $h_{0} \cdots h_{N}$ real random variables distributed according to

$$
Z_{V, N}^{-1} \exp \left(-\sum_{1}^{N} e\left(h_{i}-h_{i-1}\right)\right) \delta\left(h_{0}\right) \delta\left(h_{N}\right) \delta\left(\sum_{i} h_{i}-V\right) \prod_{i} d h_{i},
$$

where $Z_{V, N}$ is a normalization factor and $V=\lambda N^{2}$. If $e(\cdot)$ verifies hypothesis $(5 a)$ or (5b) then the mean profile of the droplet verifies asymptotically as $N \rightarrow \infty$

$$
\frac{d}{d x} \frac{d}{d \tan \Theta(x)} \frac{\beta \sigma_{A B}(\Theta(x))}{\cos \Theta(x)}=\mathrm{cst},
$$

which is the shape of the droplet corresponding to the Wulff construction.

Proof. As $N \rightarrow \infty$, we have

$$
\frac{\beta \sigma_{A B}(\Theta(x))}{\cos (\Theta(x))}=-\log \int e^{-e\left(t\left[(1-2 x) c_{\lambda}\right]+y\right)+(1-2 x) c_{\lambda} y} d y,
$$

where $c_{\lambda}$ has been defined after Eq. (8) and

$$
\tan \Theta(x)=t\left[(1-2 x) c_{\lambda}\right] .
$$

By integration by parts, we get

$$
\frac{d}{d \tan \Theta(x)} \frac{\beta \sigma_{A B}(\Theta(x))}{\cos \Theta(x)}=(1-2 x) c_{\lambda},
$$


which leads therefore to

$$
\frac{d}{d x} \frac{d}{d \tan \Theta(x)} \frac{\beta \sigma_{A B}(\Theta(x))}{\cos \Theta(x)}=-2 c_{\lambda} .
$$

That this equation corresponds to the Wulff construction may be shown by a simple variational calculation with constraint $[2,3]$.

Proposition 2. Let a sessile drop on a wall be described by $h_{0} \cdots h_{N}(N \geqq 2)$ real random variables distributed according to

$$
\Xi_{V}^{-1} e^{-\beta\left(\sigma_{B W}-\sigma_{A W}\right) N} \exp \left(-\sum_{1}^{N} e\left(h_{i}-h_{i-1}\right)\right) \delta\left(h_{0}\right) \delta\left(h_{N}\right) \delta\left(\sum_{i} h_{i}-V\right) \prod_{i} d h_{i},
$$

where

$$
\Xi_{V}=\sum_{N \geq 2} e^{-\beta\left(\sigma_{B W}-\sigma_{A W}\right) N} Z_{V, N} .
$$

If e $(\cdot)$ verifies hypothesis $(5 a)$ or $(5 b)$, then the contact angle $\Theta_{0}$ of the droplet verifies asymptotically as $V \rightarrow \infty$ the generalized Young equation:

$$
\cos \Theta_{0} \sigma_{A B}\left(\Theta_{0}\right)-\left.\sin \Theta_{0} \frac{d \sigma_{A B}}{d \Theta}\right|_{\Theta=\Theta_{0}}=\sigma_{A W}-\sigma_{B W}
$$

Proof. The contact angle $\Theta_{0}$ for a given $N$ is given by

$$
\operatorname{tg} \Theta_{0}=t(c)
$$

where $c$ satisfies

$$
\frac{I(c)}{c^{2}}=\frac{V}{N^{2}}
$$

and $N$ is to be optimized. Let us now compute the left-hand side of (21). We get

$$
\beta \cos \Theta_{0} \cdot \sigma_{A B}\left(\Theta_{0}\right)-\left.\beta \sin \Theta_{0} \cdot \frac{d \sigma_{A B}}{d \Theta}\right|_{\Theta_{0}}=-\log \int e^{-e\left(\tan \Theta_{0}+y\right)+c y} d y+c t(c) .
$$

On the other hand, the most probable length $N$ of the droplet verifies asymptotically

$$
\beta\left(\sigma_{A W}-\sigma_{B W}\right)=-\frac{d}{d N} \log Z_{V, N} \approx-\frac{d}{d N} N \int_{0}^{1} \log z_{0}\left((1-2 x) c_{N}\right) d x .
$$

We now use

$$
\begin{aligned}
\frac{d}{d c} \log z_{0}(c)= & -c \frac{d}{d c} t(c), \quad \frac{d c_{N}}{d N}=2 c_{N}\left(2 \frac{V}{N^{2}}-\frac{1}{2} t\left(c_{N}\right)\right)^{-1}-\frac{V}{N^{3}}, \\
& \int_{0}^{1} t\left((1-2 x) c_{N}\right) x d x=-\frac{V}{N^{2}},
\end{aligned}
$$

together with integration by parts, to obtain:

$$
\int_{0}^{1} \log z_{0}\left((1-2 x) c_{N}\right) d x=\log z_{0}\left(c_{N}\right)+c_{N}\left(t\left(c_{N}\right)-4 V / N^{2}\right)
$$


and

$$
\frac{d}{d N} N \int_{0}^{1} \log z_{0}\left((1-2 x) c_{N}\right) d x=\log z_{0}\left(c_{N}\right)+c_{N} t\left(c_{N}\right)
$$

so that the optimal $N$, or equivalently the optimal $c$, will verify

$$
\beta\left(\sigma_{A W}-\sigma_{B W}\right)=-\log \left(\int e^{-e(t(c)+x)+c x} d x\right)+c t(c) .
$$

This equation gives us the value of $c$, let us denote it $c^{*}$, which corresponds to the most probable length $N$. For this droplet, we get the desired equation

$$
\cos \Theta_{0} \sigma_{A B}\left(\Theta_{0}\right)-\left.\sin \Theta_{0} \frac{d}{d \Theta} \sigma_{A B}(\Theta)\right|_{\Theta_{0}}=\sigma_{A W}-\sigma_{B W}
$$

Along the same line of reasoning, one can check that this most probable length for the droplet is characterized by relative fluctuations which go to zero as the droplet becomes macroscopic.

This concludes our analysis of one sessile droplet on a wall. It remains to show that a gas of droplets will concentrate into one macroscopic droplet. This is the content of the next section.

\section{A Gas of Droplets and the Generalized Young Equation}

We consider an interface above a wall, $h_{0}=0, h_{1} \geqq 0, \ldots, h_{j} \geqq 0 \cdots h_{L}=0$. Phase $A$ is above the interface and phase $B$ is between the interface and the wall $h=0$. The energy cost of the interface is chosen as

$$
\sum_{1}^{L}\left(J_{2}+J_{1} e\left(h_{i}-h_{i-1}\right)\right)
$$

with $e(x)$ as before, and $e(0)=0$ so that $J_{2}$ is the energy per unit length of a horizontal interface. The interaction energy of the wall with phases $A$ and $B$ is taken respectively as $J_{A W}$ and $J_{B W}$ per unit length. The corresponding Boltzmann factor at inverse temperature $\beta$ is then

$$
\exp \left\{-\beta \sum_{1}^{L}\left(J_{2}+J_{1} e\left(h_{i}-h_{i-1}\right)\right)\right\} \prod_{1}^{L}\left(\exp \left(-\beta J_{B W}\right)+\delta\left(h_{i}\right) \exp \left(\beta\left(J_{2}-J_{A W}\right)\right)\right) \text {. }
$$

We wish to describe a situation where phases $A$ and $B$ are in coexistence: $A$ occupies the main part of the bulk, but phase $B$, along the wall of length $L$, also occupies a macroscopic "volume" (area) $\lambda L^{2}$ with $\lambda$ small. We thus have a canonical constraint

$$
V=\sum_{0}^{L} h_{i}=\lambda L^{2}
$$

The question is the number, size and shape of droplets of the phase $B$ on the wall. A droplet of length $l$ and volume $v$ is defined by a piece of interface

$$
h_{x}=0, \quad h_{i}>0, \quad x<i<x+l \quad h_{x+l}=0,
$$


with

$$
\sum_{i=x}^{x+l} h_{i}=v
$$

As $L \rightarrow \infty$ with $V=\lambda L^{2}$, we shall prove that the bulk of phase $B$ is concentrated in one droplet, the remainder of the wall being unaffected by the volume constraint.

Let

$$
\begin{aligned}
\Xi_{V, L}= & \int_{0}^{\infty} d h_{0} \cdots \int_{0}^{\infty} d h_{L} \delta\left(V-\Sigma h_{i}\right) \delta\left(h_{0}\right) \delta\left(h_{L}\right) . \\
& \cdot \exp \left\{-\beta \sum_{1}^{L}\left(J_{2}+J_{1} e\left(h_{i}-h_{i-1}\right)\right)\right\} \\
& \cdot \prod_{1}^{L}\left\{\exp \left(-\beta J_{B W}\right)+\delta\left(h_{i}\right) \exp \left(\beta\left(J_{2}-J_{A W}\right)\right)\right\} .
\end{aligned}
$$

We have

$$
\begin{aligned}
\Xi_{V, L}= & e^{-L \beta J_{A W}} \sum_{n \geq 1} \int d v_{1} \cdots d v_{n} \delta\left(V-\Sigma v_{p}\right)_{0 \leq x_{1}<y_{1} \leq \cdots \leq x_{n}<y_{n} \leq L} \\
& \cdot \prod_{p=1}^{n} e^{-\beta\left(J_{2}+J_{B W}-J_{A W}\right)\left(y_{p}-x_{p}-1\right)} Z_{v_{p}, y_{p}-x_{p},},
\end{aligned}
$$

where $x_{p}$ and $y_{p}$ denote the first and last point of the $p^{\prime}$ th droplet and

$$
Z_{v, l}=\int_{0}^{\infty} d h_{0} \cdots \int_{0}^{\infty} d h_{l} \delta\left(v-\sum_{1}^{l} h_{i}\right) \delta\left(h_{0}\right) \delta\left(h_{l}\right) \exp \left(-\beta J_{1} \sum_{1}^{l} e\left(h_{i}-h_{i-1}\right)\right) .
$$

We shall call a droplet "large" when its length $l$ and volume $v$ satisfy

$$
\frac{v}{l}>(\log L)^{1+\varepsilon}
$$

where $\varepsilon>0$ is fixed. We then have:

Proposition 3. As $L \rightarrow \infty$ with $V=\hat{\lambda} L^{2}$, the probability that there is more than one large droplet is less than $\exp \left(-\frac{1}{2}(\log L)^{1+\varepsilon}\right)$.

Proof. Let $V_{0}$ and $L_{0}$ be the total volume of the large droplets, and $n_{0}$ their number.

Let

$$
\Xi_{V . L}=\sum_{n_{0} \geq 1} \Xi_{V, L}^{n_{0}}
$$

where $\Xi_{V, L}^{n_{0}}$ is defined as the contribution with $n_{0}$ large droplets. We have

$$
\begin{aligned}
\Xi_{V, L}^{1}= & \sum_{L=L_{1}+L_{0}+L_{2}} \int d V_{0} d V_{1} d V_{2} \delta\left(V-V_{0}-V_{1}-V_{2}\right) \Xi_{V_{1}, L_{1}}^{0} \cdot Z_{V_{0}, L_{0}} \cdot \Xi_{V_{2}, L_{2}}^{0} \\
\Xi_{V . L}^{n}< & L^{n-1} \sum_{L=L_{1}+L_{0}+L_{2}} \int d V_{0} d V_{1} d V_{2} \delta\left(V-V_{0}-V_{1}-V_{2}\right) . \\
& \cdot \Xi_{V_{1} L_{1}}^{0} \cdot \int d v_{1} \cdots d v_{n} \delta\left(V_{0}-\Sigma v_{p}\right) \sum_{L_{0}=\Sigma l_{p}} \prod Z_{v_{p}, l_{p}} \cdot \Xi_{V_{2}, L_{2}}^{0} \cdot
\end{aligned}
$$

The factor $L^{n-1}$ bounds the number of possible positions of the large droplets. The strict inequality comes from the fact that $\Xi_{V_{1}, L_{1}}^{0} \cdot \Xi_{V_{2}, L_{2}}^{0}$ overestimates the contri- 
bution of the small drops: $L_{1}+L_{2}$ is in fact cut into $n+1$ rather than 2 pieces, which reduces the configuration space (the factor $L^{n-1}$ and the sum over $l_{1} \cdots l_{n}$ have already taken care of the choice of the position of $n-1$ intermediate intervals).

Lemma 2. Let $\tilde{Z}_{V, L}$ be defined as in (17). Then $\exists a>0, b>0$ such that $\forall v \geqq 0, l \geqq 2$, we have

$$
-l^{-1} \log \frac{\tilde{Z}_{i, l}}{\tilde{Z}_{0, l}}>a \frac{v}{l^{2}}-b l^{-1} \log (l) .
$$

Proof. Our hypothesis for $e(x)$ imply

$$
e(x)>a^{\prime}|x|-b^{\prime} \quad \forall x .
$$

Using half of $e(x)$ to bound the integration over the $h$ 's and keeping the other half, we get

$$
\tilde{Z}_{v, l}<\operatorname{cst}^{l} \exp \left(-\frac{1}{2} \beta J_{1} a^{\prime} \operatorname{Inf} \Sigma\left|x_{i}\right|\right)
$$

where the infimum is taken over the configurations satisfying the volume constraint. This infimum is achieved for a rectangular droplet, and therefore

$$
\Sigma\left|x_{i}\right| \geqq 2 \frac{v}{l}
$$

which implies

$$
-l^{-1} \log \frac{\tilde{Z}_{v, l}}{\tilde{Z}_{0, l}}>\beta J_{1} a^{\prime} \frac{v}{l^{2}}-\mathrm{cst} .
$$

This establishes the lemma for $v / l^{2}$ large or for $l$ small. We now consider $l \rightarrow \infty$ with $v / l^{2}$ bounded; we can then use Theorem 2, (see also (18)), which can be written as follows:

with

$$
-l^{-1} \log \widetilde{Z}_{v, l}=\operatorname{cst}+f\left(\frac{v}{l^{2}}\right)+O\left(l^{-1} \log l\right)
$$

$$
\begin{aligned}
& f(\lambda)=-\frac{1}{2} \int_{-1}^{1} d u \log \tilde{z}_{0}\left(u c_{\lambda}\right)+\log \tilde{z}_{0}(0), \\
& \tilde{z}_{0}(c)=\int e^{-\beta J_{1} e(t(c)+y)+c y} d y .
\end{aligned}
$$

and $c_{\lambda}$ and $t(c)$ were defined in (7), (8). We then get

$$
\begin{aligned}
f^{\prime}(\lambda) & =-\frac{1}{2} \int_{-1}^{1} u d u \tilde{z}_{0}^{-1}\left(u c_{\lambda}\right) \cdot \tilde{z}_{0}^{\prime}\left(u c_{\lambda}\right) \cdot c_{\lambda}^{\prime} \\
& =\frac{c_{\lambda}^{\prime}}{2} \int_{-1}^{1} u^{2} u d u\left(-\langle y\rangle_{0}+t^{\prime}\left(u c_{\lambda}\right)\left\langle e^{\prime}\left(t\left(u c_{\lambda}\right)+y\right)\right\rangle_{0}\right) \\
& =\frac{1}{2} c_{\lambda} c_{\lambda}^{\prime} \int_{-1}^{1} u^{2} d u t^{\prime}\left(u c_{\lambda}\right)
\end{aligned}
$$

where we have used $\langle y\rangle_{0}=0$ and integration by parts for the second term. Now 
from

we get

$$
I\left(c_{\lambda}\right)=\lambda c_{\lambda}^{2}
$$

$$
\begin{gathered}
\frac{1}{2} c_{\lambda} c_{\lambda}^{\prime} t\left(c_{\lambda}\right)=c_{\lambda}^{2}+2 \lambda c_{\lambda} c_{\lambda}^{\prime} \\
c_{\lambda}^{\prime}=\left(\frac{1}{2} l\left(c_{\lambda}\right)-2 \lambda\right)^{-1} c_{\lambda} .
\end{gathered}
$$

But $t\left(c_{\lambda}\right)=\operatorname{tg} \Theta_{0}$, where $\Theta_{0}$ is the Young contact angle. Since the droplet is convex and symmetric, $\Theta_{0}$ is larger than it would be for the triangular droplet:

$$
\operatorname{tg} \Theta_{0}>\operatorname{tg} \Theta_{0}(\text { triangle })=4 \frac{V}{L^{2}}=4 \lambda
$$

Therefore $c_{\lambda}^{\prime}>0$ and $f^{\prime}(\lambda)>$ cst $>0$. This concludes the proof of Lemma 2 with

$$
a=\inf _{0 \leqq \lambda \leqq v / l^{2}} f^{\prime}(\lambda) \text {. }
$$

We now estimate $\Xi_{V, L}^{n}$ using

$$
\prod Z_{v_{p}, l_{p}}<\prod \tilde{Z}_{v_{p}, l_{p}}<Z_{V_{0}, L_{0}}\left(\prod \frac{\tilde{Z}_{v_{p}, l_{p}}}{\tilde{Z}_{0, l_{p}}}\right) \frac{\tilde{Z}_{0, L_{p}}}{Z_{V_{0}, L_{0}}}
$$

In models where $\lambda^{-1} f(\lambda)$ is increasing in $\lambda$ (recall the definition of $f$ in (22)), such as the gaussian model, the proof can be concluded easily: there is at least one $p$, say $p=1$, such that

$$
\frac{v_{1}}{l_{1}}>\frac{V_{0}}{L_{0}}
$$

which implies of course $\left(v_{1} / l_{1}^{2}\right)>\left(V_{0} / L_{0}^{2}\right)$. We then have, if $\lambda^{-1} f(\lambda)$ is increasing,

$$
\frac{l_{1}^{2}}{v_{1}} f\left(\frac{v_{1}}{l^{2}}\right)>\frac{L_{0}^{2}}{V_{0}} f\left(\frac{V_{0}}{L_{0}^{2}}\right) \Rightarrow l_{1} f\left(\frac{v_{1}}{l_{1}^{2}}\right)>L_{0} f\left(\frac{V_{0}}{L_{0}^{2}}\right) \Rightarrow \frac{\tilde{Z}_{v_{1}, l_{1}}}{\tilde{Z}_{0, l_{1}}}<\frac{\tilde{Z}_{V_{0}, L_{0}}}{\tilde{Z}_{0, L_{0}}} \cdot e^{O\left(\log L_{0}\right)} .
$$

Using Lemma 2 for $p=2, \ldots, n$ then yields

$$
\prod Z_{v_{p}, l_{p}}<Z_{V_{0}, L_{0}} \exp \left(-\sum_{p \geq 2} a \frac{v_{p}}{l_{p}}\right)<Z_{V_{0}, L_{0}} e^{-(n-1) a(\log L)^{1+\varepsilon}}
$$

which proves Proposition 3. In the present general framework however, we do not know whether $\lambda^{-1} f(\lambda)$ is increasing. We distinguish the tall droplets as those for which

$$
a \frac{v_{p}}{l_{p}^{2}}>f\left(\frac{V_{0}}{L_{0}^{2}}\right)
$$

so that use of Lemma 2 will be enough. For the others, we use an argument inspired by the Wulff construction. Consider two such droplets $\left(v_{1}, l_{1}\right)$ and $\left(v_{2}, l_{2}\right)$ with $\left(v_{1} / l_{1}\right) \geqq\left(v_{2} / l_{2}\right)$. We shall prove 


$$
\tilde{Z}_{v_{1}, l_{1}} \tilde{Z}_{v_{2}, l_{2}}<\tilde{Z}_{v_{1}+v_{2}, l_{1}+l_{2}}\left(\frac{\tilde{Z}_{v_{2}, l_{2}}}{\tilde{Z}_{0, l_{2}}}\right)^{1 / 2}
$$

Indeed

$$
\begin{aligned}
-\log \tilde{Z}_{v_{1}, l_{1}} \tilde{Z}_{v_{2}, l_{2}} \simeq & \int_{0}^{l_{1}} \frac{\sigma\left(\Theta_{1}(x)\right)}{\cos \Theta_{1}(x)} d x+\int_{l_{1}}^{l_{1}+l_{2}} \frac{\sigma\left(\Theta_{2}(x)\right)}{\cos \Theta_{2}(x)} d x+O\left(\log l_{1}\right)+O\left(\log l_{2}\right) \\
= & \int_{0}^{l_{1}} \frac{\sigma\left(\Theta_{1}(x)\right)}{\cos \Theta_{1}(x)} d x+\int_{l_{1}}^{l_{1}+l_{2} / 2} \sigma(0)+\int_{l_{1}+l_{2} / 2}^{l_{2}} \frac{\sigma\left(\Theta_{2}(x)\right)}{\cos \Theta_{2}(x)} \\
& +\int_{l_{1}}^{l_{1}+l_{2} / 2}\left(\frac{\sigma\left(\Theta_{2}(x)\right)}{\cos \Theta_{2}(x)}-\sigma(0)\right) d x+O\left(\log l_{1}\right)+O\left(\log l_{2}\right) .
\end{aligned}
$$

The last term will provide the damping factor in inequality (23). The second and third term correspond to an interface which bounds a volume $v_{2} / 2+l_{2} / 2 \cdot h_{\max }(2)$. For the first term, we have (see Fig. 1)

$$
\int_{0}^{l_{1}} \frac{\sigma\left(\Theta_{1}(x)\right)}{\cos \Theta_{1}(x)} d x>\int_{0}^{a_{1}} \frac{\sigma\left(\Theta_{1}(x)\right)}{\cos \Theta_{1}(x)} d x+\int_{a_{1}}^{b_{1}} \sigma(0) d x+\int_{b_{1}}^{l_{1}} \frac{\sigma\left(\Theta_{1}(x)\right)}{\cos \Theta_{1}(x)} d x,
$$

because $\sigma(\Theta) / \cos \Theta$ is increasing in $\Theta$. Indeed

$$
\begin{aligned}
\frac{\sigma(\Theta)}{\cos \Theta} & =-\log \tilde{z}_{0}(c) \text { with } t(c)=\operatorname{tg} \Theta, \\
\tilde{z}_{0}(c) & =\int e^{-\beta J_{1} e(y)+c(y-t(c))} d y, \\
\frac{d}{d c} \frac{\sigma(\Theta)}{\cos \Theta} & \left.=-\langle y-t(c)\rangle_{0}+c t^{\prime}(c)=c\left(t^{\prime}(c)\right)\right\rangle 0 .
\end{aligned}
$$

We now adjust $a_{1}$ and $b_{1}$ so that the new interface above $\left(0, l_{1}\right)$ bounds a volume

$$
v_{1}+\frac{v_{2}}{2}-\frac{l_{2}}{2} h_{\max }(2)
$$

and meets the interface above $\left(l_{1}, l_{1}+l_{2}\right)$ corresponding to the second and third term in (24).

Fig. 1.

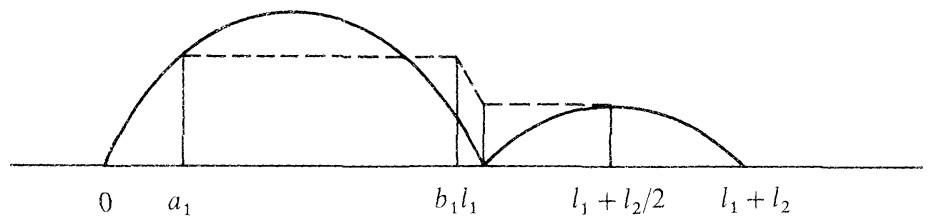

The resulting interface above $\left(0, l_{1}+l_{2}\right)$ bounds a volume $v_{1}+v_{2}$, but is not optimal. Therefore

$$
\begin{aligned}
-\log \tilde{Z}_{v_{1}, l_{1}} \tilde{Z}_{v_{2}, l_{2}}> & \int_{0}^{l_{1}+l_{2}} \frac{\sigma\left(\Theta_{1+2}(x)\right)}{\cos \Theta_{1+2}(x)} d x \\
& +\int_{l_{1}}^{l_{1}+l_{2} / 2}\left(\frac{\sigma\left(\Theta_{2}(x)\right)}{\cos \Theta_{2}(x)}-\sigma(0)\right) d x+O\left(\log l_{1}\right)+O\left(\log l_{2}\right) .
\end{aligned}
$$


Exponentiating this inequality gives

$$
\begin{aligned}
\tilde{Z}_{v_{1}, l_{1}} \tilde{Z}_{v_{2}, l_{2}} & <\tilde{Z}_{v_{1}+v_{2}, l_{1}+l_{2}}\left(\frac{\tilde{Z}_{v_{2}, l_{2}}}{\tilde{Z}_{0, l_{2}}}\right)^{1 / 2} e^{O\left(\log l_{1}\right)+O\left(\log l_{2}\right)} \\
& <\tilde{Z}_{v_{1}+v_{2}, l_{1}+l_{2}} \exp \left(-\frac{1}{2} a \frac{v_{2}}{l_{2}}+O\left(\log l_{1}\right)+O\left(\log l_{2}\right)\right) .
\end{aligned}
$$

Iterating this procedure yields

$$
\prod \tilde{Z}_{v_{p}, l_{p}}<\exp \left(-a \Sigma^{\prime} \frac{v_{p}}{l_{p}}-\frac{1}{2} a \Sigma^{\prime \prime} \frac{v_{p}}{l_{p}}\right) \tilde{Z}_{V_{0}^{\prime \prime}, L_{0}^{\prime \prime}}
$$

where $\Sigma^{\prime}$ is the sum over tall droplets $a v_{p} / l_{p}^{2}>f\left(V_{0} / L_{0}^{2}\right)$, and $\Sigma^{\prime \prime}$ the sum over the others, and

$$
\begin{array}{ll}
V_{0}^{\prime \prime}=\Sigma^{\prime \prime} v_{p}, & L_{0}^{\prime \prime}=\Sigma^{\prime \prime} l_{p}, \\
V_{0}^{\prime}=\Sigma^{\prime} v_{p}, & L_{0}^{\prime}=\Sigma^{\prime} l_{p} .
\end{array}
$$

We then have

$$
\begin{aligned}
& \prod Z_{v_{p}, l_{p}}<Z_{V_{0}, L_{0}} \exp \left(-a \Sigma^{\prime} \frac{v_{p}}{l_{p}}-\frac{1}{2} a \Sigma^{\prime \prime} \frac{v_{p}}{l_{p}}-L_{0}^{\prime \prime} f\left(\frac{V_{0}^{\prime \prime}}{L_{0}^{\prime \prime 2}}\right)\right) \\
& \quad \exp \left(L_{0} f\left(\frac{V_{0}}{L_{0}^{2}}\right)+\Sigma O\left(\log l_{p}\right)\right) .
\end{aligned}
$$

We now use

$$
L_{0} f\left(\frac{V_{0}}{L^{2}}\right)<L_{0}^{\prime \prime} f\left(\frac{V_{0}^{\prime \prime}}{L_{0}^{\prime \prime 2}}\right)+\operatorname{cst}\left(\frac{V_{0}}{L_{0}^{2}}\right)\left(L_{0}^{\prime}+\frac{V_{0}^{\prime}}{L_{0}}\right)
$$

where $\operatorname{cst}\left(V_{0} / L_{0}^{2}\right)$ is bounded in terms of $f^{\prime}(x)$ with $x<V_{0} / L_{0}^{2}$. Using $v_{p} / l_{p}>(\log L)^{1+\varepsilon}$, we get

$$
\prod Z_{v_{p}, l_{p}}<Z_{V_{0}, L_{0}} \exp \left(-(n--1) \frac{a}{2}(\log L)^{1+\varepsilon} \frac{a}{2} \Sigma^{\prime} \frac{v_{p}}{l_{p}}+\operatorname{cst}\left(\frac{V_{0}}{L_{0}^{2}}\right)\left(L_{0}^{\prime}+\frac{V_{0}^{\prime}}{L_{0}}\right)\right) .
$$

From $v_{p} / l_{p}^{2}>A$, we get $v_{p} / l_{p}>A l_{p}$ and $l_{p}<\left(V_{0} / A\right)^{1 / 2}$. Therefore

$$
\begin{gathered}
\prod Z_{v_{p}, l_{p}}<Z_{V_{0}, L_{0}} \exp \left(-(n-1) \frac{a}{2}(\log L)^{1+\varepsilon}-\left(\frac{a}{4} A-c\left(\frac{V_{0}}{L_{0}^{2}}\right)\right) L_{0}^{\prime}\right) \\
\cdot \exp \left(-\left(\frac{a}{4}\left(\frac{L_{0}^{2} A}{V_{0}}\right)^{1 / 2}-\operatorname{cst}\left(\frac{V_{0}^{\prime}}{L_{0}^{2}}\right)\right) \frac{V_{0}^{\prime}}{L_{0}}\right) .
\end{gathered}
$$

This shows that $A$ can be chosen such that

$$
\prod Z_{p_{p}, l_{p}}<Z_{V_{0}, L_{0}} e^{-(n-1)(\log L)^{1+\epsilon^{*}}} .
$$

This concludes the proof of Proposition 3.

We now have only one large droplet, and we know from Sect. 3 that its shape obeys the Wulff construction. There remains to determine the value of $V_{0} / L_{0}^{2}$, or 
equivalently to prove that the contact angle obeys the modified Young equation. We have

$$
\Xi_{V, L}^{1}=\sum_{L=L_{1}+L_{0}+L_{2}} \int d V_{0} d V_{1} d V_{2} \delta\left(V-V_{0}-V_{1}-V_{2}\right) \Xi_{V_{1}, L_{1}}^{0} Z_{V_{0}, L_{0}} \Xi_{V_{2}, L_{2}}^{0} .
$$

If we first perform the integral over the volumes, we see that

$$
\begin{aligned}
Z_{V_{0}, L_{0}} & =\exp \left(-L_{0} f\left(\frac{V_{0}}{L^{2}}\right)+O\left(\log L_{0}\right)\right) \\
& =\exp \left(-L_{0} f\left(\frac{V}{L_{0}^{2}}\right)+\frac{V_{1}+V_{2}}{L_{0}} f^{\prime}\left(\frac{V}{L_{0}^{2}}\right)+O\left(\log L_{0}\right)\right) .
\end{aligned}
$$

On the other hand, the volume dependence of $\Xi_{V_{1}, L_{1}}^{0}$ (and $\Xi_{V_{2}, L_{2}}^{0}$ ) could be estimated by looking at the distribution function of $\Sigma h_{i}$ without volume constraint, in the partial wetting situation in which we are interested, characterized by a finite mean size of droplets. One would find $\left\langle V_{1}\right\rangle \approx L_{1}$ and $\left\langle\left(V_{1}-\left\langle V_{1}\right\rangle\right)^{2}\right\rangle \approx L_{1}$ because $V_{1}$ is the sum of $O\left(L_{1}\right)$ volumes of independent droplets. Therefore a Gaussian distribution

$$
\exp \left(-\frac{\left(V_{1}-\left\langle V_{1}\right\rangle\right)^{2}}{2 \sigma^{2} L_{1}}\right)
$$

will cut off the integral at

$$
V_{1} \sim\left\langle V_{1}\right\rangle+O\left(L_{1}^{1 / 2}\right)
$$

before the volume dependence of $Z_{V_{0}, L_{0}}$ can come into the game. Therefore

$$
\Xi_{V, L} \sim \sum_{L_{0}} \Xi_{L-L_{0}} Z_{V, L_{0}}
$$

where $\Xi_{L-L_{0}}$ is the grand canonical partition function (no volume constraint). We then have

$$
\log \Xi_{L-I_{0}} \cdot Z_{V . L_{0}}=\left(L-L_{0}\right) \beta \sigma_{A W}+L_{0} \beta \sigma_{B W}+\int_{0}^{L_{0}} \frac{\beta \sigma_{A B}(\Theta(x))}{\cos \Theta(x)} d x+O(\log L),
$$

and we are back to the classical variational problem, which we know yields the modified Young equation. The sum over $L_{0}$ will be peaked around the optimal value, with a fluctuation less than $L^{1 / 2}$. In order that this optimal value be less than $L$, we need $V / L^{2}$ less than some constant which would vanish at the wetting transition.

\section{Appendix}

Lemma 3. Let $e(x)$ satisfy hypothesis $(5 a)$ or $(5 b)$. Then $\exists 0<c_{\max } \leqq \infty$ such that

$$
t(c)=\frac{\int x e^{-e(x)+c x} d x}{\int e^{-e(x)+c x} d x}
$$

and 


$$
I(c)=\frac{1}{2} \int_{0}^{c} c^{\prime} t\left(c^{\prime}\right) d c^{\prime}
$$

are continuous increasing functions of $c \in\left[0, c_{\max }[\right.$ and

$$
\begin{array}{lll}
t(c) \rightarrow+\infty & \text { as } & c \rightarrow c_{\max } \\
I(c) \rightarrow+\infty & \text { as } & c \rightarrow c_{\max } .
\end{array}
$$

Remark. Our hypothesis on $e(x)$ was stated in [1, Theorem 1 and Remark], it guarantees that the surface tension $\sigma(\Theta)$ is given by

$$
\sigma(\Theta)=-\cos \Theta \log \left(\int d x e^{-e(x+t)+c x}\right),
$$

where $t=\tan \Theta$ and $c$ is the solution of

$$
\int x e^{-e(x+t)+c x} d x=0 .
$$

Proof. The proof is straightforward, with $c_{\max }=\infty$ for $(5 \mathrm{a})$ and $c_{\max }=c_{0}$ for (5b).

Lemma 4. Let $\vec{X}_{n} \in \mathbb{R}^{d}, n \in \mathbb{N}, j=1, \ldots, n$ be centered independent random variables whose distribution functions have densities which are uniformiy continuous, uniformly in $j$, n. Let

$$
\begin{gathered}
\vec{Y}_{n}=\left(Y_{n}^{1}, \ldots, Y_{n}^{d}\right)=n^{-1 / 2} \sum_{j=1}^{n} \vec{X}_{j, n}, \\
\Gamma_{n}^{\alpha \beta}=\left\langle Y_{n}^{\alpha} Y_{n}^{\beta}\right\rangle=n^{-1} \sum_{j=1}^{n}\left\langle X_{j n}^{\alpha} X_{j n}^{\beta}\right\rangle .
\end{gathered}
$$

Suppose that the $d x d$ matrix $\Gamma_{n}$ converges as $n \rightarrow \infty$ to a positive definite matrix $I$, and that for some integer $k \geqq 3$ and some $\delta>0$

$$
\limsup n^{-1} \sum_{j=1}^{n}\left\langle\left(\vec{X}_{j n}^{2}\right)^{k / 2+\delta}\right\rangle<\infty
$$

Then the random variable $\vec{Y}_{n}$ has a density $f_{n}(\vec{Y})$ such that

$$
f_{n}(\vec{Y})=n_{\Gamma_{n}}(\vec{Y})\left(1+\sum_{\gamma=1}^{k-2} \frac{P_{\gamma n}(\vec{Y})}{n^{\gamma / 2}}\right)+O\left(n^{-(k-2) / 2}\right),
$$

where $n_{\Gamma_{n}}(\vec{Y})$ is the density of the Gaussian measure of covariance $\Gamma_{n}$ and $P_{\gamma n}(\vec{Y})$ are polynomials of total degree $3 \gamma-1$ in $Y^{1} \ldots Y^{d}$ whose coefficients are bounded in modulus from above uniformly in $n$.

Proof. For $d=1$, or for random variables $\vec{X}_{j n}$ which do not vary with $n$, the result is standard $[4,5,6]$. The proof here is essentially the same.

Acknowledgements. The authors wish to thank the CNRS, the Universite de l'Etat à Mons, and the Communauté Française de Belgique for financial support which made their collaboration possible. They also wish to thank Ch.-Ed. Pfister for interesting comments. 


\section{References}

1. De Coninck, J., Dunlop, F.: Partial to complete wetting: A microscopic derivation of the young relation. J. Stat. Phys. 47, 827-849 (1987)

2. Landau, L. D., Lifshitz, E. M.: Statistical physics, Vol. 1. Oxford: Pergamon Press 1980

3. van H. Beijeren, Nolden, I.: The roughening transition. In Current topics in physics, Schommers and von Blankenhagen (eds.). Berlin, Heidelberg, New York: Springer 1987

4. Bhattacharya, R. N., Ranga, Rao, R.: Normal approximation and asymptotic expansions Wiley series in Probability and Mathematical Statistics, p. 199. New York: Wiley 1976

5. Feller, W.: An introduction to probability theory and its applications, Vol. 2, p. 535, New York: Wiley 1971

6. Petrov, V. V.: Sums of independent random variables, pp. 175, 206. Berlin, Heidelberg, New York: Springer 1975

7. Durrett, R. T., Iglehart, D. L.: Weak convergence to Brownian meander and Brownian excursion. Ann. Probab. 5, 117-129 and 130-135 (1977)

Communicated by A. Jaffe

Received May 27, 1988; in revised form August 19, 1988 
